\title{
ESTUDIO PRELIMINAR SOBRE LA DETERMINACIÓN DE ELEMENTOS TRAZA EN CERVEZAS VENEZOLANAS POR ICP-OES
}

\author{
Eunice Marcano, Clara Gómez, Zully Benzo y Jorge Laine* \\ Centro de Química, Instituto Venezolano de Investigaciones Científicas, Apartado 20632, Caracas 1020-A, Venezuela
}

Recebido em 14/5/09; aceito em 1/10/09; publicado na web em 2/3/10

\begin{abstract}
PRELIMINARY STUDY ON THE DETERMINATION OF TRACE ELEMENTS IN VENEZUELAN BEERS BY ICP-OES. The levels of several chemical elements were determined simultaneously in ten different beers of the Venezuelan market by ICP-OES. With the exception of chromium, beer does not provide important amounts of nutritional oligoelements. The average chromium content found $(33 \mu \mathrm{g} / \mathrm{L})$ is higher than one reported for USA's beer $(10 \mu \mathrm{g} / \mathrm{L})$ but smaller than another reported for Brazilian beer $(66 \mu \mathrm{g} / \mathrm{L})$. The average percentage of chromium RDA by beer consumption found for Venezuela (6.3\%) is similar to that reported for Belgium (5\%). Regarding toxic elements, one of the samples contained an elevated amount of aluminium, probably dissolved from the aluminium can.
\end{abstract}

Keywords: beer analysis; chromium in beer; ICP-OES.

\section{INTRODUCCIÓN}

La importancia de la presencia de metales disueltos en cerveza puede abarcar diferentes aspectos: i) como influyente en la preparación industrial de la misma. ${ }^{1}$ Por ejemplo, niveles altos de cobre y cinc pueden interferir negativamente retardando el proceso de producción, ${ }^{2}$ y se ha referido que el hierro acelera el deterioro de la cerveza. ${ }^{3}$ ii) En el aspecto toxicológico, por la posible presencia de ciertos elementos como el $\mathrm{Al}, \mathrm{Cd}, \mathrm{Pb}, \mathrm{Hg}$, los cuales el organismo humano no puede regular. ${ }^{4}$ iii) En el aspecto nutricional, por la presencia de ciertos elementos referidos como esenciales o oligoelementos tales como $\mathrm{Cr}, \mathrm{Fe}, \mathrm{Mn}, \mathrm{Cu}, \mathrm{Zn}$, etc. ${ }^{5,6}$ Estos dos últimos aspectos pueden tener importancia en Venezuela, país que está entre los mayores consumidores de cerveza en el mundo, ${ }^{7}$ siendo el mayor en toda América tal como se refiere en la Tabla 1.

Tabla 1. Mayores consumidores de cerveza (litros per cápita, 2005)

\begin{tabular}{lcc}
\hline 1 & República Checa & 160.5 \\
2 & Irlanda & 127.4 \\
3 & Alemania & 109.9 \\
4 & Austria & 105.8 \\
5 & Bélgica & 98.6 \\
6 & Reino Unido & 95.7 \\
7 & Dinamarca & 92.5 \\
8 & Eslovenia & 92.4 \\
9 & Australia & 87.8 \\
10 & Venezuela & 83.3 \\
11 & Estados Unidos & 82.8 \\
12 & España & 82.1 \\
13 & Finlandia & 81.7 \\
14 & Hungría & 80.1 \\
15 & Nueva Zelanda & 77.3 \\
\hline
\end{tabular}

Desde que Schwarz y Merzt probaron que la presencia de cromo es esencial en el cuerpo humano para el metabolismo de la glucosa, ${ }^{8}$ se ha demostrado que la deficiencia de ese elemento puede ser prevenida por una ingesta apropiada del mismo. ${ }^{9} \mathrm{El}$ cromo es escaso en

*e-mail: jlaine@ivic.gov.ve la mayoría de los alimentos, siendo la levadura una de las fuentes más ricas en este elemento. ${ }^{10}$ Probablemente el cromo ingresa en la cerveza a través de las materias primas, especialmente de la levadura, y posiblemente también por liberación de cromo de los tanques y tuberías de acero inoxidable durante el proceso de fabricación. La asimilación de cromo por medio de la ingesta moderada de cerveza podría considerarse positiva desde el punto de vista nutricional. ${ }^{11} \mathrm{De}$ hecho, en la revista Brauwelt, especializada en cerveza, los beneficios fisiológicos de la misma como bebida luego de la realización de alguna competencia deportiva exhaustiva ha sido reportado. ${ }^{6}$

Se han usado varias técnicas analíticas para determinar cuantitativamente la presencia de elementos importantes en la cerveza, tanto desde los puntos de vista industrial, nutricional y toxicológico. ${ }^{12-22}$ La técnica analítica utilizada en el presente trabajo es la espectrometría de emisión atómica por plasma acoplado inductivamente (ICP-OES), la cual permite una precisa determinación de un buen número de elementos con la ventaja de poder realizar análisis simultáneo secuencial, con límites de detección especialmente favorables para elementos refractarios (hasta 1000 veces más bajos que con espectrometría de absorción atómica con llama, FAAS), con interferencias no espectrales pequeñas, y con un rango linear amplio. Sus principales desventajas son el alto costo de los equipos, el alto ruido de fondo, y las interferencias ocasionadas por las bandas de grupos hidroxilo.

El objetivo de este trabajo es el uso de la técnica ICP-OES para determinar elementos traza nutricionales $(\mathrm{Cr}, \mathrm{Cu}, \mathrm{Fe}, \mathrm{Mn}, \mathrm{Zn})$ en cervezas venezolanas, con un énfasis especial en la consideración de este producto como una fuente nutricional de cromo; determinando simultáneamente también otros elementos nutricionales $(\mathrm{Ca}$, $\mathrm{K}, \mathrm{Mg}, \mathrm{Na}, \mathrm{P}$ ), así como también la posible presencia de elementos potencialmente tóxicos.

\section{PARTE EXPERIMENTAL}

\section{Aparatos}

Fue usado un espectrómetro simultáneo ICP, marca Perkin-Elmer, modelo OPTIMA 3000, de la Perkin Elmer Corporation, Norwalk, U.S.A.. Para la desgasificación de las cervezas, se utilizó un baño de ultrasonido, marca Cole Palmer modelo 8851 de la compañía Cole Palmer Instrument Company, Chicago, U.S.A.. 


\section{Reactivos}

En este estudio de usó agua ultrapura (18 $\mathrm{M} \Omega \mathrm{cm})$ obtenida de un equipo desionizador marca Millipore modelo Milli-Q de la compañía Millipore Corporation, Billerica, U.S.A.. Los patrones usados para la cuantificación fueron preparados por dilución de las soluciones multielementales: 11355 ICP multi element standard IV de la Merck, Darmstadt, Alemania, y de N930-218 multielement atomic spectrocopy standard, de la Perkin Elmer Corporation, Norwalk, U.S.A.

La incorporación del lantano como patrón interno a muestras y estándares se realizó mediante dilución de la solución de 1 g/L de lantano de la Aldrich Company Inc., Milwaukee, U.S.A..

\section{Muestras}

Se utilizaron un total de 10 cervezas, de las cuales 4 estaban enlatadas y el resto en botellas de vidrio; del total 9 eran cervezas rubias tipo Pilsen y 1 denominada ("Cerveza E") era negra. Ocho cervezas eran producidas en Venezuela y 2 eran importadas procedentes de Holanda ("Cerveza D Lata" y "Cerveza D Botella"). Del total de cervezas, 2 estaban contenidas en botellas retornables ("Cerveza B Botella" y "Cerveza F Botella"). Los envases de las cervezas restantes eran desechables. Todas las muestras fueron adquiridas en establecimientos comerciales. Cervezas de la misma marca y tipo pero con diferentes envases están referidas con la misma letra (A, B, etc).

\section{Preparación de las muestras para el análisis}

Las cervezas fueron desgasificadas por ultrasonido, para evitar inestabilidad en el plasma, e introducidas directamente en el espectrómetro de ICP. Se trabajó con 5 réplicas de cada muestra.

\section{Procedimiento del análisis}

Una vez desgasificadas las cervezas, las mismas (un total de 10) fueron introducidas en el espectrómetro y los elementos fueron determinados cuantitativamente, en forma simultánea por ICP-OES. Los elementos mayoritarios fueron: potasio, calcio, magnesio, fósforo y sodio y los elementos trazas: aluminio, cromo, cobre, hierro, manganeso y cinc. Estos elementos fueron medidos en las longitudes de onda mostradas en la Tabla 2. Pruebas preliminares con el patrón multielemental permitieron verificar que los elementos tóxicos $(\mathrm{Cd}$, $\mathrm{Pb}$ y $\mathrm{Hg}$ ) estaban debajo de los límites de detección (LD) de la técnica en dichas muestras (LD de Cd, $\mathrm{Pb}$ y $\mathrm{Hg}: 1,8 ; 39,0$ y $3,8 \mu \mathrm{g} / \mathrm{L}$ respectivamente).

Para la cuantificación se aplicó el método de calibración externa con estandarización interna para compensar la influencia del etanol. El estándar interno usado fue el lantano, agregado a igual concentración a patrones acuosos y muestras $(1,00 \mathrm{mg} / \mathrm{L})$.

El rango de patrones utilizado para los elementos mayoritarios (Ca, Mg, P, Na , K) fue: 0-200 ppm (mg/L) y para los trazas: 0-300 $\mathrm{ppb}(\mu \mathrm{g} / \mathrm{L})$.

Se optimizaron automáticamente las condiciones de trabajo del equipo (potencia, flujo del nebulizador y altura de observación) con una rutina del software del equipo (Directed Search algorithm). En la Tabla 2 se resumen las condiciones de trabajo de este análisis.

\section{RESULTADOS Y DISCUSIÓN}

Los resultados de las concentraciones de los elementos analizados en las cervezas son mostrados en la Tabla 3. Los errores en los valores de las 5 medidas de cada muestra fueron siempre menores al $5 \%$. Estos resultados no muestran relación uniforme ni con el tipo de
Tabla 2. Condiciones instrumentales y longitudes de onda $(\lambda)$ utilizadas

\begin{tabular}{lc}
\hline Parámetro & Valor \\
\hline Potencia & $1300 \mathrm{Watts}$ \\
Flujo del nebulizador & $0.5 \mathrm{~L} / \mathrm{min}$ \\
Flujo del plasma & $15 \mathrm{~L} / \mathrm{min}$ \\
Flujo auxiliar & $2.0 \mathrm{~L} / \mathrm{min}$ \\
Velocidad de bomba & $1.0 \mathrm{~mL} / \mathrm{min}$ \\
Altura de observación & $15 \mathrm{~mm}$ \\
Corrección de fondo & Manual \\
Tipo de nebulizador & Meinhard \\
Elemento & \\
$\mathrm{Al}$ & $\lambda(\mathrm{nm})$ \\
$\mathrm{Ca}$ & 396.152 \\
$\mathrm{Cr}$ & 396.847 \\
$\mathrm{Cu}$ & 206.149 \\
$\mathrm{Fe}$ & 324.754 \\
$\mathrm{Mn}$ & 238.204 \\
$\mathrm{Zn}$ & 260.569 \\
$\mathrm{Mg}$ & 213.856 \\
$\mathrm{Na}$ & 279.079 \\
$\mathrm{~K}$ & 589.592 \\
$\mathrm{P}$ & 766.491 \\
$\mathrm{La}$ & 213.618 \\
\hline
\end{tabular}

envase (vidrio retornable, vidrio desechable o lata), ni con el tipo o procedencia de cerveza (Pilsen o negra, nacional o importada). En el caso del cromo, el valor promedio de concentración (33 $\mu \mathrm{g} / \mathrm{L}$, Tabla 3$)$, es más alto que el reportado por Anderson y Bryden, ${ }^{11}$ para cervezas de EE.UU. (10 $\mu \mathrm{g} / \mathrm{L})$, pero más bajo que el reportado para cervezas brasileñas $(66 \mu \mathrm{g} / \mathrm{L})$ por Matsushige y De Oliveira. ${ }^{16}$

Los valores de hierro (rango 10 hasta $36 \mu \mathrm{g} / \mathrm{L}$ ) y cobre (10 hasta $36 \mu \mathrm{g} / \mathrm{L}$ ), fueron aceptables comparándolos con niveles máximos permisibles de $200 \mu \mathrm{g} / \mathrm{L}$ de cada metal encontrados en la literatura. ${ }^{13}$

Aunque se ha mencionado en la literatura, ${ }^{23}$ la posible contaminación de ciertos cationes como el sodio por parte de residuos de los detergentes (hidróxido de sodio) usados en la etapa de limpieza de los envases retornables, se puede observar que, tomando el ejemplo de una cerveza enlatada y una embotellada retornable de la misma marca ( "Cerveza B Lata” y " Cerveza B Botella”, Tabla 3), la concentración de sodio no difiere en forma importante entre estos dos tipos de muestra, por lo que se puede inferir que en este caso los agentes limpiantes no han dejado huella apreciable en estas muestras.

En cuanto al posible riesgo de consumo de algún elemento tóxico (como podría ser el aluminio), se puede generalizar, observando los resultados de los microelementos (Tabla 3), que el riesgo de absorción de elementos tóxicos ingiriendo cerveza puede ser similar o menor que al consumir agua potable. En efecto, una muestra de agua tomada de un bebedero público fue analizada obteniéndose valores de: $\mathrm{Al}=152 \mu \mathrm{g} / \mathrm{L}, \mathrm{Cu}=56 \mu \mathrm{g} / \mathrm{L}, \mathrm{Fe}=130 \mu \mathrm{g} / \mathrm{L}, \mathrm{Mn}=101 \mu \mathrm{g} / \mathrm{L}, \mathrm{Zn}=1526$ $\mu \mathrm{g} / \mathrm{L}, \mathrm{Cr}<10 \mu \mathrm{g} / \mathrm{L}$. El alto contenido de $\mathrm{Zn}$ en esta muestra se debe probablemente a las tuberías galvanizadas que normalmente se usan en las conexiones de las aguas servidas. Por otra parte, una muestra de agua mineral comercial, embotellada en plástico, tenia un contenido de $\mathrm{Al}=4 \mu \mathrm{g} / \mathrm{L}, \mathrm{Zn}=52 \mu \mathrm{g} / \mathrm{L}$, y trazas menores de $10 \mu \mathrm{g} / \mathrm{L}$ para los demás oligoelementos.

Cabe destacar el relativo alto contenido de $\mathrm{Al}$ en solo una de las cervezas analizadas: $225 \mu \mathrm{g} / \mathrm{L}$ (muestra D en lata, importada de Holanda), lo cual puede ser debido a la disolución del aluminio de la lata por parte de la cerveza, debido a defectos en la capa plástica de 
Tabla 3. Resultados de los análisis ICP-OES

\begin{tabular}{|c|c|c|c|c|c|c|c|c|c|c|c|c|}
\hline Muestra & Envase & $\begin{array}{c}\mathrm{Al} \\
(\mu \mathrm{g} / \mathrm{L})\end{array}$ & $\begin{array}{c}\mathrm{Cr} \\
(\mu \mathrm{g} / \mathrm{L})\end{array}$ & $\begin{array}{c}\mathrm{Cu} \\
(\mu \mathrm{g} / \mathrm{L})\end{array}$ & $\begin{array}{c}\mathrm{Fe} \\
(\mu \mathrm{g} / \mathrm{L})\end{array}$ & $\begin{array}{c}\mathrm{Mn} \\
(\mu \mathrm{g} / \mathrm{L})\end{array}$ & $\begin{array}{c}\mathrm{Zn} \\
(\mu \mathrm{g} / \mathrm{L})\end{array}$ & $\begin{array}{c}\mathrm{Ca} \\
(\mathrm{mg} / \mathrm{L})\end{array}$ & $\begin{array}{c}\mathrm{Mg} \\
(\mathrm{mg} / \mathrm{L})\end{array}$ & $\begin{array}{c}\mathrm{Na} \\
(\mathrm{mg} / \mathrm{L})\end{array}$ & $\begin{array}{c}\mathrm{K} \\
(\mathrm{mg} / \mathrm{L})\end{array}$ & $\begin{array}{c}\mathrm{P} \\
(\mathrm{mg} / \mathrm{L})\end{array}$ \\
\hline A & $\mathrm{L}$ & 14 & 24 & 24 & 11 & 122 & 10 & 23 & 85 & 69 & 382 & 119 \\
\hline A & B & 60 & 17 & 36 & 36 & 142 & 49 & 20 & 85 & 49 & 407 & 137 \\
\hline B & $\mathrm{L}$ & 10 & 10 & 24 & 16 & 85 & 10 & 18 & 67 & 62 & 357 & 105 \\
\hline B & $\mathrm{B}^{\mathrm{R}}$ & 80 & 104 & 10 & 10 & 10 & 67 & 18 & 71 & 62 & 341 & 116 \\
\hline $\mathrm{C}$ & $\mathrm{L}$ & 29 & 41 & 27 & 21 & 59 & 87 & 39 & 54 & 12 & 281 & 81 \\
\hline $\mathrm{C}$ & B & 82 & 91 & 27 & 26 & 93 & 62 & 40 & 55 & 15 & 255 & 80 \\
\hline $\mathrm{D}$ & $\mathrm{L}$ & 225 & 13 & 26 & 24 & 45 & 28 & 17 & 35 & 13 & 274 & 70 \\
\hline $\mathrm{D}$ & B & 24 & 10 & 26 & 22 & 63 & 27 & 16 & 40 & 23 & 308 & 76 \\
\hline $\mathrm{E}$ & $\mathrm{B}$ & 10 & 11 & 16 & 30 & 37 & 32 & 24 & 29 & 21 & 223 & 56 \\
\hline $\mathrm{F}$ & $\mathrm{B}^{\mathrm{R}}$ & 10 & 10 & 69 & 39 & 34 & 26 & 15 & 30 & 23 & 302 & 69 \\
\hline Promedio & & 55 & 33 & 23 & 24 & 69 & 39 & 23 & 55 & 35 & 313 & 91 \\
\hline
\end{tabular}

$\mathrm{L}=$ envase de lata, $\mathrm{B}=$ botella de vidrio, ${ }^{\mathrm{R}}=$ retornable

Tabla 4. Aporte nutricional de la cerveza en Venezuela

\begin{tabular}{lcccc}
\hline Elemento & $\begin{array}{c}\text { Concentración } \\
\text { mg/L }\end{array}$ & $\begin{array}{c}\text { Aporte de } \\
\text { Cerveza }^{\mathrm{b}} \\
\text { mg/día.persona }\end{array}$ & $\begin{array}{c}\text { RDA }^{\mathrm{c}} \\
\mathrm{mg} / \text { dia } \\
\text { persona }\end{array}$ & $\begin{array}{c}\text { \% Aporte } \\
\text { (promedio) }^{\mathrm{d}}\end{array}$ \\
\hline $\mathrm{Cr}$ & 0.033 & 0.0076 & 0.12 & 6.3 \\
$\mathrm{Cu}$ & 0.023 & 0.0053 & 2 & 0.3 \\
$\mathrm{Fe}$ & 0.024 & 0.0055 & 10 & 0.0 \\
$\mathrm{Mn}$ & 0.069 & 0.0159 & 2 & 0.8 \\
$\mathrm{Zn}$ & 0.039 & 0.0090 & 15 & 0.1 \\
$\mathrm{Ca}$ & 23 & 5.3 & 1600 & 0.3 \\
$\mathrm{~K}$ & 313 & 72.0 & 4000 & 1.8 \\
$\mathrm{Mg}$ & 55 & 12.6 & 400 & 3.2 \\
$\mathrm{P}$ & 91 & 20.9 & 1000 & 2.1
\end{tabular}

${ }^{\mathrm{a} C}$ Concentraciones promedio obtenidas en este trabajo. ${ }^{\mathrm{b}}$ Cantidad que aporta la cerveza en base al consumo diario per cápita en Venezuela (0.23 L/día.persona, calculado de la Tabla 1). ${ }^{c}$ Mínima cantidad diaria recomendada por el Instituto Nacional de Nutrición, Venezuela. 'Porcentaje del RDA (Recommende Daily Alowance) aportado por la cerveza en Venezuela (Nota: este valor debe ser mucho mayor si se refiere solo a la población que realmente consume cerveza, la cual debe ser una pequeña fracción de la población total).

recubrimiento interior que llevan las latas de aluminio, o a condiciones extremas (elevada temperatura y agitación) durante el transporte y almacenamiento.

Conociendo el consumo per cápita promedio diario de cerveza en Venezuela (Tabla 1), el valor promedio de concentración de cromo en las muestras analizadas ( $33 \mu \mathrm{g} / \mathrm{L})$, y el requerimiento de cromo mínimo recomendado por día para un adulto, $0.12 \mathrm{mg}$, puede tenerse una idea del porcentaje de ese requerimiento que aportaría el consumo per cápita de cerveza. Este aporte $(6.3 \%$, Tabla 4) resulta similar al valor reportado por Robberecht et al. para el caso de Bélgica (5\%). ${ }^{10}$ Cabe resaltar en la Tabla 4 que, con la excepción del cromo, la cerveza no aporta nutricionalmente un porcentaje importante de los demás elementos traza analizados, pero si puede aportar cantidades importantes de algunos de los otros elementos nutrientes ( $\mathrm{K}, \mathrm{Mg}, \mathrm{y} \mathrm{P})$.

Es importante resaltar que el valor real de esos porcentajes calculados deben ser considerablemente mayores, teniendo en cuenta que el valor per cápita toma en cuenta a toda la población, y sólo una fracción de ésta es la que realmente consume cerveza.

\section{CONCLUSIONES}

La determinación en forma simultánea de los niveles de varios elementos químicos en 10 diferentes cervezas del mercado venezolano por ICP-OES demuestra que, con excepción del cromo, la cerveza no aporta cantidades importantes de oligoelementos nutrientes. El porcentaje del requerimiento dietético recomendado ( $R D A$ en inglés) de cromo por el consumo de cerveza en Venezuela es similar al reportado para Bélgica. En cuanto a elementos tóxicos, los resultados sugieren que la probabilidad de encontrar aluminio disuelto en la cerveza es mayor en envases de aluminio que en envases de vidrio.

\section{REFERENCIAS}

1. Leubolt, R.; Huber, C.; Urban, A.; Puspok, J.; Mitt. Ost. Getr. Inst. 1992, 46,80 .

2. Martins, S. M.; Como fabricar cerveja, Editora Ícone: São Paulo, 1987.

3. Kaneda, H.; Kano, Y.; Koshino, S.; Ohya Nishiguchi, H.; J. Agr. Food Chem. 1992, 40, 2102.

4. Carter, D. D.; Fernandus, Q. ; J. Chem. Educ. 1979, 56, 490.

5. Cerutti, G.; Ciappellano, S.; Casartelli, A.; Imbottigliamento 1993, 16, 69.

6. Piendl, A.; Brauwelt 1990, 130, 370.

7. Carroll, C.; National Geographic 2006, 210, 24.

8. Schwarz, K.; Merzt, W.; Arch. Biochem. Biophys. 1959, 85, 292.

9. Jeejeebhoy, K.; Chu, R.; Marliss, E.; Greenberg, J.; Bruce, A.; Am. J. Clin. Nutr. 1977, 30, 531 .

10. Robberecht, H.; van Schoor, O.; Deelstra, H. S.; J. Food Sci. 1984, 49, 300 .

11. Anderson, R.; Bryden, N.; J. Agr. Food Chem. 1983, 31, 308.

12. Borriello, R.; Sciaudone, G.; Atom. Spectrosc. 1980, 1, 131.

13. Dubiel, L.; Prz. Fermentacyjny I Owocowo-Warzywny. 1992, 36, 4.

14. Finoli, C.; Vecchio, A.; Volonterio, G.; Gigliotti, C.; Ind. delle Bevande 1990, $19,1$.

15. Kuthanova, E.; Cernohorsky, T.; Matrka, M.; Cesk. Hygiena 1993, 38, 36.

16. Matsushige, I.; Oliveira, E.; Food Chem. 1993, 47, 205.

17. Senften, H.; Brauerei- und Getraenke-Rundschau 1993, 104, 107.

18. Sharpe, F. R.; Williams, D. R.; J. Am. Soc. Brew. Chem. 1995, 53, 85.

19. Wagner, H. P.; McGarrity, M. J.; J. Am. Soc. Brew. Chem. 1990, $48,1$.

20. Ward, A.; Elemental analysis of beer using the inductively coupled plasma, Jarrel-Ash Division, Fischer Scientific Co., 1977.

21. Weiner J. P.; Taylor, L.; J. Institute of Brewing 1969, 75, 195.

22. William, D. R.; Brewer 1996, 82, 102.

23. Roesicke, J.; Renkamp, L.; Brauwelt 1990, 130, 408. 